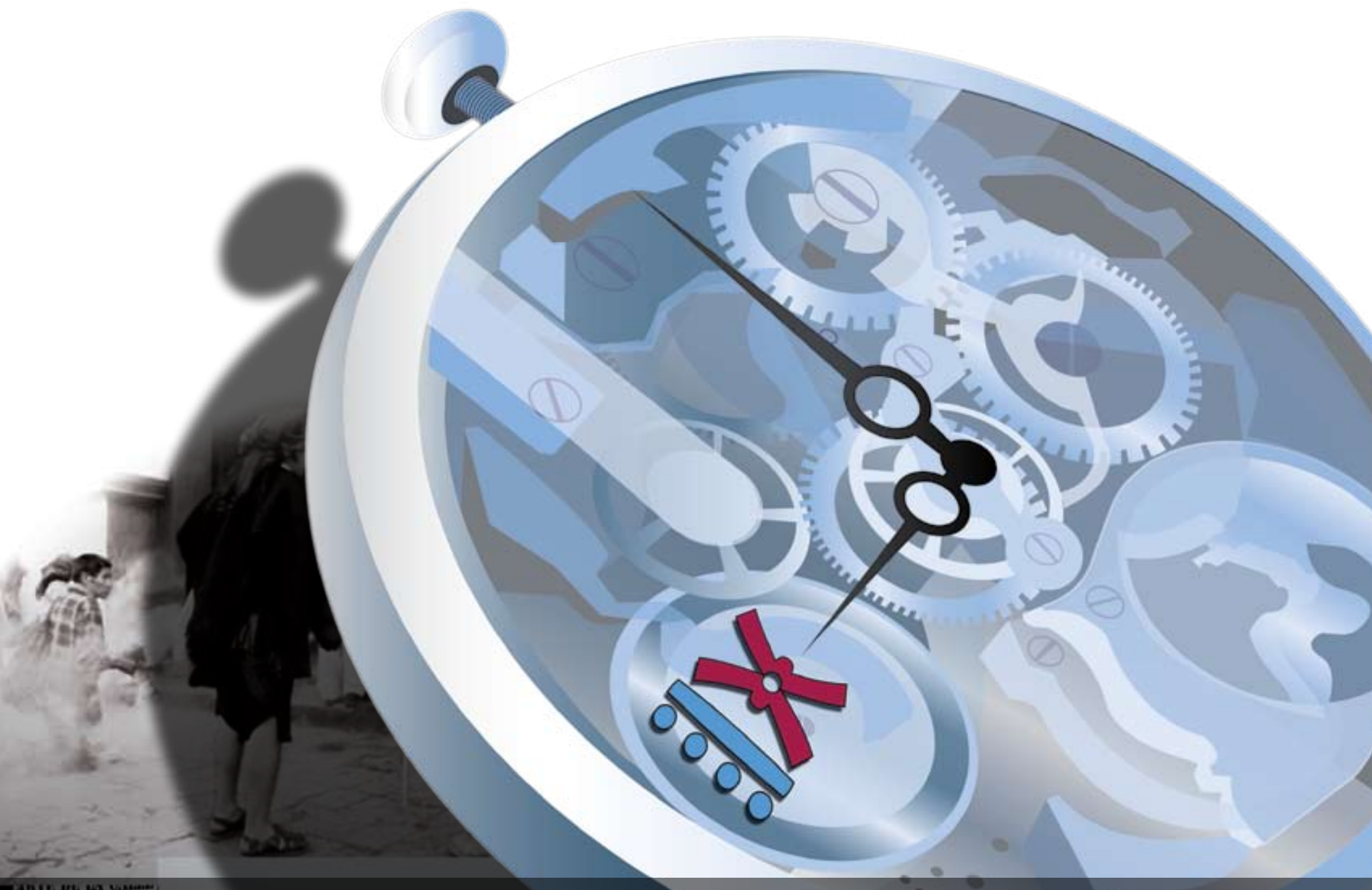

ᄀ $9^{\circ}$ C ONGRESO 92 CENTROAMERICANO DE HISTORIA
ISSN 1409-469X

Fecha de recepción: 15 de mayo 2008 Fecha de aceptación: 30 de mayo 2008

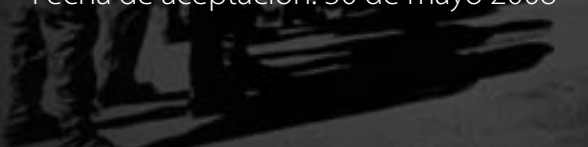

Redes sociales, caficultores y poder local. El caso del municipio de Santiago de María en el oriente de El Salvador, 1874-1920

Miembros del Consejo Editorial:

Dr. Ronny Viales, Dr. Juan José Marín

Editores Técnicos:

Allan Fonseca, Andrés Cruz, Gabriela Soto
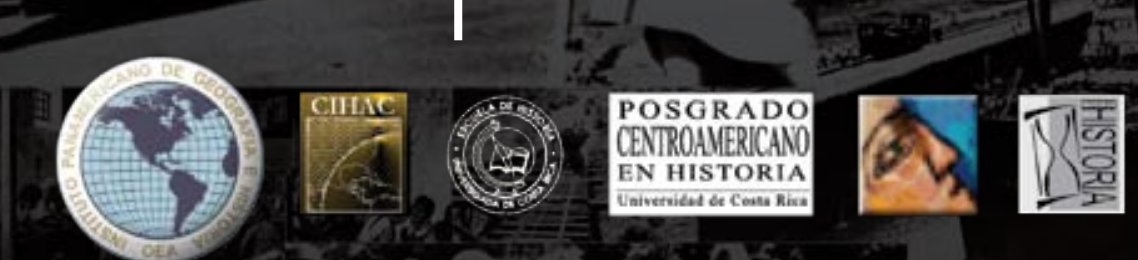


\section{Redes sociales, caficultores y poder local. El caso del municipio de Santiago de María en el oriente de El Salvador, 1874-1920 ${ }^{1}$.}

oscarcamposlara@hotmail.com Historiador. Consultor y Director de la revista electrónica Hacer historia en El Salvador

1 Esta ponencia se desprende del trabajo de tesis para optar al grado de licenciado en Historia, titulado: Cafetaleros de Santiago de María y la formación de un poder micro-regional en el departamento de Usulután, 1894-1917. Universidad Tecnológica de El Salvador, diciembre 2006. 


\section{Introducción.}

La ponencia que se presenta es fruto de una investigación surgida en la inquietud por conocer la geografía económica, la sociedad y poder cafetaleros en El Salvador desde la experiencia local; tomando en consideración que en la configuración y auge de la economía cafetalera participaron personas de todos los niveles sociales, con los matices según la diversidad de contextos locales.

Precisamente es esta diversidad en la que inserta este caso, partiendo que existe un desbalance historiográfico donde prevalecen estudios realizados sobre el occidente, por ser la zona cafetalera tradicional, la más importante y activa económicamente del país; razón por la cual se pretende hacer una excepción al poner atención en la zona oriental salvadoreña. El único antecedente de matices locales al respecto, es el estudio sobre la historia regional del café realizado por Aldo Lauria, quien realiza una periodización y establece variantes regionales en el proceso de expansión y consolidación de la economía cafetalera en la conjunción los siglos XIX y XX. Dicha periodización plantea tres etapas: un periodo inicial entre 1850-60 donde la producción se centra en pocos municipios localizados principalmente en la zona occidental ${ }^{2} \mathrm{y}$ en los departamentos centrales de La Libertad y San Salvador, con la participación de agricultores de todos los niveles sociales quienes empiezan a cultivar en los alrededores de las áreas urbanas y en los terrenos ejidales y comunales circundantes. El éxito de los pioneros conduce a una segunda etapa (18701890) marcada por la expansión a otras zonas aptas a lo largo del país. En esta etapa se verifica la extensión del número de propiedades, de agricultores y de tamaño en los 8 departamentos donde atraviesa de oriente a occidente la cadena volcánica activa salvadoreña; ocupando los terrenos 2 La zona occidental de El Salvador está constituida por los departamentos de Santa Ana, Sonsonate y Ahuachapán, en el complejo volcánico de la sierra Apaneca-Lamatepec, la extensión más grande del parque cafetalero salvadoreño. 
ejidales y comunales vacantes, baldíos y coberturas de bosque virgen en dichas alturas fértiles ${ }^{3}$.

Una tercera etapa (hacia el final de la $1^{\text {a }}$ Guerra Mundial) está marcada por la consolidación cuyo camino recorre una primera crisis donde la producción y expansión se estanca, dando luego signos de recuperación tras la Primera Guerra Mundial, duplicándose el parque cafetalero y experimentando el fenómeno de la concentración de tierras a raíz de la anterior crisis internacional y la complejidad local del crédito.

A partir de este planteamiento, es necesario profundizar en el campo de los actores sociales que formaron parte de este proceso, ya que suele conocerse más los resultados que a los actores y sus causas. De acuerdo con Lauria, es importante abandonar los mitos sobre la formación y funcionamiento de elites en los cuales se ha simplificado o extrapolado la noción de elite explotadora y progresista frente a un campesinado explotado o ignorante. En su artículo "Vinculando lo local con lo nacional" nos invita a reconsiderar a estos y otros sujetos y a sus categorías sociales como actores más complejos y dinámicos, tomando en cuanta las escalas de sus proyecciones personales y colectivas, sean campesinos o de la elite y no simplemente cono agentes de la economía cafetalera ${ }^{4}$.

Debido a la expansión temprana del café frente a otros puntos del país y por ubicarse la mayor extensión de suelo cafetalero entre los departamentos de Santa Ana, Sonsonate y Ahuachapán existe la noción del "occidente cafetalero". Esto ha conllevado a una preferencia académica por

3 Aldo Lauria. Historia regional del café en El Salvador. Revista de Historia No.38, julio-diciembre de 1998. San José: Universidad de Costa Rica) p.10.

$4 \quad$ Aldo Lauria y Leigh Binford, (editores). Landscapes of truggle: pilitics, society and community in El Salvador. (Pittsburg: University of Pittsburg Press, 2004). 
esta sección del territorio salvadoreño sobre el cual se han producido trabajos muy importantes en torno al poder y su resistencia, la tenencia de la tierra y otros similares.

Por su parte, la expansión y desarrollo del café en el oriente presenta algunas diferencias. Las dos principales ciudades, San Miguel y Usulután, se encuentran en valles bajos y cálidos no aptos para el café, y a varios kilómetros de las fincas que se encuentran en las faldas del complejo volcánico Tecapa-Chinameca, lugar donde emerge una nueva zona geo-económica de importancia nacional donde los municipios de Chinameca, Jucuapa, (departamento de San Miguel) Santiago de María y Berlín (departamento de Usulután) se convierten en las ciudades cafetaleras de oriente. Otra diferencia a considerar, es la baja densidad poblacional y escasa población indígena en esta zona, sumado a la fundación de nuevos municipios en el último cuarto del siglo XIX, situación que contrasta con el occidente densamente poblado y mayoritariamente indígena desde tiempos antiguos hasta la actualidad.

Podemos pensar entonces en una frontera parcialmente abierta, disponible y solamente ejidal, aunque no por mucho tiempo, pues la fundación de una decena de municipios -entre ellos Santiago de María- se produce pocos años antes y después de los decretos de extinción de tierras comunales, ejidales para su distribución a título de propiedad privada (1882-1883) situación que favorece el establecimiento de brazos y capitales para el desarrollo cafetalero.

Por lo tanto, los fenómenos estudiados parten de esta variante local, se sitúan entre la fundación del municipio en 1874 hasta la $3^{\text {a }}$ etapa planteada por Lauria y su resultado representa un matiz en la expansión y consolidación de la economía y sociedad cafetalera en El Salvador. 
La ponencia entonces, consiste en un resumen básico de la investigación mencionada, en la cual se establecerá la identificación de redes sociales articuladas en el municipio de Santiago de María, lugar donde se configura una de las elites locales más sobresalientes en la entonces república cafetalera de El Salvador. El trabajo se estructura en primer lugar con las redes económicas que se refieren a las estrategias y mecanismos de configuración de un grupo de medianos y grandes caficultores, beneficiadores y exportadores; fenómeno paralelo al tejido de redes sociales que crean y cohesionan a una elite cafetalera con alcances geográficos estratégicos. Ambas redes posibilitan la creación y relación de redes políticas que se cristalizan en el control de la municipalidad y en la articulación de un proyecto portuario acorde a los intereses cafetaleros.

\section{Redes económicas: inmigración, capitales y estrategias.}

Señales de expansión cafetalera.

Cuando se realizó en 1874 el trazo urbano para ubicar al nuevo pueblo de Santiago de María, una de las primeras líneas tiradas atravesaba la propiedad de la señora Cirila Rivera que se hallaba cultivada de café y platanar ${ }^{5}$, desconociendo cuántos de los primeros vecinos pudieron tener propiedades cultivadas como la de la señora Rivera en la misma década, pero hay indicios no haber sido la única. Los registros de la década siguiente hacen referencia a numerosos cafetales pertenecientes a algunos vecinos de la localidad, como el Alcalde en 1880, Silverio Iglesias, quien solicitó el título de su huatal cultivado con 1,600 árboles de cafée. Tres años antes, la

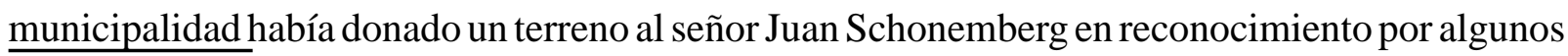
5 Archivo Municipal de Santiago de María (AMSM a partir de este momento). Libro de Actas Municipales del año de 1874 . Sesión del $1^{\circ}$ de junio.

6 AMSM. Libro de Actas Municipales del año de 1880. Sesión del 11 se octubre. 
servios (no especificados en la fuente) que realizó para la comunidad, de forma que lo dedicara al repasto de su ganado a que su terreno estaba acotado con cafetales 7 . En ese mismo año (1877) se hace referencia de una finca de café propiedad del Presbítero Fernando Araujo, mientras que los agricultores Sebastián Galliano, Agustín Gotuzo y el citado Schonemberg solicitaron a la alcaldía la cesión de un solar urbano para construir sus casas ${ }^{8}$. De este modo es claro que desde la fundación del municipio el café estaba siendo cultivado en diversas escalas por vecinos locales y extranjeros recién asentados.

A partir de la década de 1880 se dispone con una mayor cantidad de referencias que apuntan a determinarla como el inicio de la expansión del cultivo de café en el municipio de Santiago de María. Este fenómeno se produce en el aumento de las exportaciones de café, las cuales lograron superar a las producidas por el añil a inicios de la década, se duplicaron a inicios de 1890 y se triplican al final del siglo XIX. Además de los primeros actores a los que se ha hecho mención, se cuenta con la presencia de otras personas de diversas procedencias que realizaron adquisiciones importantes tales como fincas de café. Los "protocolos de predios rústicos" disponibles y las apreciaciones de la memoria municipal, son las principales referencias con que se cuentan para sostener el planteamiento.

La colonia extranjera que reside en Santiago de María para 1882 se compone de los mencionados señores Schonemberg, Galiano y Gotuzzo, además de la presencia de Ambrosio Canessa (Italia), Casimiro Donnadieu (Francia) y los presbíteros Pedro Poch y Francisco Lasplazas (españoles) $)^{9}$. De sus primeras propiedades no se sabe más que la ubicación de ellas en las zonas $7 \quad$ AMSM. Libro de Actas Municipales del año de 1877. Sesión del $1^{\circ}$ de marzo.

8 Idem. Sesión del 6 de mayo.

9 AMSM. Libro de Actas Municipales de 1882. Sesión del 24 de octubre. 
próximas al emplazamiento urbano, tales como los cerros Oromontique y volcán de Tecapa. Para 1890 se registra en las mismas memorias el crecimiento de dicha actividad, permitiendo a la municipalidad pasar a un nivel del control y aprovechamiento del cultivo para aumentar los ingresos municipales. Se había señalado la necesidad de levantar un primer censo de los cosecheros de café en el municipio, a fin de exigir impuestos por quintal ${ }^{10}$, así como también se instó a que se establecieran dos guardias que vigilaran la exportación del café de la jurisdicción para garantizar la seguridad ${ }^{11}$, pues esta actividad auguraba mayores ingresos con los cuales se pretendían llevar a cabo obras públicas necesarias en un municipio en pleno crecimiento.

El estado de la expansión en la última década del siglo XIX.

Hacia esta fecha, varios agricultores han constituido fincas de considerable extensión y como patrimonios de los caficultores pioneros de Santiago de María. Los Protocolos de predios rústicos, ejecución de herencias y compras registradas en los Libros de Registro de Propiedad y Raíz en la primera década del siglo XX son útiles para rastrear algunos de estos patrimonios, los cuales se exponen en el siguiente cuadro.

10 Ibíd. Sesión del 29 de enero de 1892. Sin embargo, ningún tipo de censo cafetalero fue encontrado hasta un documento del año de 1922.

11 AMSM. Libro de Actas Municipales de 1891. Sesión del 4 de febrero. 
Indexaciones: Repositorio de Revistas UCR, DIALNET, Latindex, REDALYC Directorio y recolector de recursos digitales del Ministerio de Cultura de España, Directory of Open Access Journals. Diálogos Revista Electrónica de Historia ISSN 1409-469X. Número especial 2008. Dirección web: http://historia.fcs.ucr.ac.cr/dialogos.htm

\begin{tabular}{|c|c|c|c|}
\hline \multicolumn{4}{|c|}{ Cuadro 1. Fincas de café en Santiago de María hacia finales del siglo XIX. } \\
\hline Fincas principales & Formas de adquisición & Fincas adyacentes & Fuentes \\
\hline $\begin{array}{l}\text { Santa Ana. Gral. Jaime Ávila } \\
\text { y sucesión. } 80 \mathrm{mz} \text { al } \mathrm{N} \text { de la } \\
\text { población. }\end{array}$ & $\begin{array}{l}\text { Compras realizadas antes } \\
\text { de } 1884 \text { y ninguna hasta la } \\
\text { ejecución de herencia, } 1911\end{array}$ & $\begin{array}{l}\text { Ambrosio Canessa } \\
\text { Ester C. de Homar } \\
\text { J. Schonemberg }\end{array}$ & $\begin{array}{l}\text { AMSM. Protocolo } \\
\text { de títulos de predios } \\
\text { rústicos. Año de } 1884 \text {. } \\
\text { partidas } 5 \text { y } 6 \text {. }\end{array}$ \\
\hline Montecristo, San Pedro y Los & Las dos primeras por compra & Pedro Poch, & Idem. Año de 1899, \\
\hline $\begin{array}{l}\text { Diamantes. Sebastian Galliano } \\
\text { y Sra. Paula Bexio de Galliano. } \\
3 \text { fincas } 25,15 \text { y } 23 \mathrm{mz} \text {. Dos con } \\
\text { cafetos fructificando y otra con } \\
60,000 \text { cafetos }\end{array}$ & $\begin{array}{l}\text { a Pedro Poch, la última por } \\
\text { compra al Cnel. José Dolores } \\
\text { Nuila. Sin fechas. }\end{array}$ & $\begin{array}{l}\text { Manuel Calvo } \\
\text { Francisco Herrera } \\
\text { Simón Sol } \\
\text { Evaristo Iglesias } \\
\text { Patricio Chavarría }\end{array}$ & partida No.2. \\
\hline $\begin{array}{l}\text { Juan Schonemberg y sucesión. } \\
126 \mathrm{mz} \text { en Las Flores, } 64 \mathrm{mz} \text { en } \\
\text { Marquesado, } 10 \mathrm{mz} \text { en Las Playas }\end{array}$ & $\begin{array}{l}\text { Sin datos. La finca en el } \\
\text { Marquesado valorada en } \\
\text { 12,000 pesos. }\end{array}$ & $\begin{array}{l}\text { Agustín Gotuzzo, } \\
\text { Jaime Ávila, } \\
\text { Simón Sol }\end{array}$ & $\begin{array}{l}\text { CNR-Usulután. } \\
\text { Libro de Registro } \\
\text { No.2, partidas 203- } \\
\text { 205. Año de } 1898\end{array}$ \\
\hline $\begin{array}{l}\text { Adrián Ortiz y sucesión. } \\
\text { 10-15mz Cerro El Tigre }\end{array}$ & No se tiene más datos & Ninguno. & $\begin{array}{l}\text { Idem. Libro 16, } \\
\text { partidas 257-262, año } \\
\text { de } 1909 .\end{array}$ \\
\hline $\begin{array}{l}\text { "La Paz", de Carlos Ezeta. 30-40 } \\
\text { mz. Con maquinaria de secado, } 2 \\
\text { pajas de agua y } 2 \text { casas }\end{array}$ & $\begin{array}{l}\text { Sin datos. Valorado en } 20,000 \\
\text { pesos. }\end{array}$ & $\begin{array}{l}\text { Prudencio Llach } \\
\text { Salvador Samayoa }\end{array}$ & $\begin{array}{l}\text { Idem. Libro 5, } \\
\text { partidas 225-229. Año } \\
\text { de } 1901\end{array}$ \\
\hline
\end{tabular}




\begin{tabular}{|c|c|c|c|}
\hline $\begin{array}{l}\text { La Veneciana, de Ambrosio } \\
\text { Canessa. 100-140mz en los } \\
\text { suburbios. Maquinaria y casa. }\end{array}$ & $\begin{array}{l}\text { Sin datos. Desde las } \\
\text { referencias en } 1880 \text {, ninguna } \\
\text { compra registrada hasta su } \\
\text { embargo en } 1909 \text {. }\end{array}$ & $\begin{array}{l}\text { Luis Duke } \\
\text { Jaime Ávila } \\
\text { J. Schonemberg }\end{array}$ & $\begin{array}{l}\text { Tdem. Libro 22, } \\
\text { partida 52. Año de } \\
\text { 1909. }\end{array}$ \\
\hline $\begin{array}{l}\text { Miramar, de Simón Sol. } 125 \mathrm{mz} \text {. } \\
\text { Compuesta de varias porciones. } \\
\text { Una casa. Cerro El Tigre }\end{array}$ & $\begin{array}{l}\text { Compras durante la década de } \\
1880 \text { (cuadro 1). Heredada en } \\
1900 .\end{array}$ & $\begin{array}{l}\text { Sebastián Galliano } \\
\text { Fco. Lasplazas } \\
\text { C. Cocchella }\end{array}$ & $\begin{array}{l}\text { Tdem. Libro 6, partida } \\
\text { 54. Año de } 1900 .\end{array}$ \\
\hline $\begin{array}{l}\text { La Catalana, del Pbto. Francisco } \\
\text { Lazplazas en el Cerro El Tigre. } \\
\text { 73mz aproximadamente. }\end{array}$ & $\begin{array}{l}\text { Sin datos. Desde referencias } \\
\text { en la década de } 1880 \text {, sin } \\
\text { registro de compras hasta su } \\
\text { venta en } 1907\end{array}$ & $\begin{array}{l}\text { Victoriano Ruíz } \\
\text { Prudencio Llach } \\
\text { (comprador) }\end{array}$ & $\begin{array}{l}\text { Idem. Libro 19, } \\
\text { partidas 54-55. Año } \\
\text { de 1907. }\end{array}$ \\
\hline
\end{tabular}

Es importante considerar en este cuadro a las fincas adyacentes de las cuales se cuenta con más información en referencias posteriores. Otros cafetaleros que comienzan a figurar en el municipio son Mauricio Meardi, Luis Manent, los sanmigueleños Dr. Enrique Rómulo Rosales y Felipe Macay, y algunos miembros de la familia Araujo como Rosendo y el Dr. Miguel del mismo apellido, todos ellos presentes al menos desde $1890^{12}$, contando con algunas referencias de adquisiciones en distintos puntos de la Sierra Tecapa hacia los inicios del siglo XX. Estas informaciones nos permiten concluir que durante la década de 1880 se inicia la expansión del cultivo de café en Santiago de María.

12 AMSM. Libro de Actas Municipales de 1890. Sesión del 4 de junio. Las personan mencionadas aportaron cantidades no pequeñas de dinero para colaborar en la construcción de una pila pública y la compra de terreno para ensanchar la ciudad. 
En lo que respecta a la extinción de tierras ejidales, las pocas referencias encontradas permiten inferir que la disposición de tierras estuvo al alcance de los solicitantes, sobre todo en zonas alejadas del emplazamiento urbano, tales como las fronteras con Jucuapa, Santa Elena y California donde se repartió una buena cantidad de tierras solicitadas, especialmente en el Cerro El Tigre. Esto permite pensar además en un panorama complicado para los numerosos interesados que se avecindaron en el municipio a partir de la década de 1890, pues la abundancia de tierras es bastante relativa. Las mejores (o las más solicitadas) zonas ya estaban ocupadas por las fincas de Schonemberg, Galeano, Gotuzzo, Canessa, Ávila, Lasplazas y Sol, cercanas a los caminos, con mayor disponibilidad de agua por su cercanía al valle donde se encuentra la ciudad.

Factores claves en las redes económicas: inmigración local y extranjera.

"Ciudad del trabajo y de cuantiosa migración”, eran palabras que describían al municipio por parte de algunos estudios de geografía publicados en las primeras décadas del siglo XX. "Es uno de los principales centros cafetaleros", asfirmaba Miguel Escamilla en la Geografía Económica de El Salvador ${ }^{13}$. En la misma época dijo Santiago Barberena: la suavidad de su clima, la belleza del paisaje y más que todo la fertilidad suma de su suelo atrajeron cuantiosa migración ávida de trabajo y se formaron como por encanto valiosas fincas de café" ${ }^{14}$. Así también se refirió Pedro Fonseca en 1915 sobre Santiago de María como "la ciudad del trabajo, centro agrícola y

13 Miguel Escamilla. Geografía económica de la república de El Salvador. (San Salvador: Imprenta La Unión, 1908) p.41.

14 Barberena, Santiago. Monografia del Departamento de Usulután. (San Salvador: Imprenta Nacional, 1909) p.23. 
comercial intenso. La inmigración extranjera ha contribuido al desarrollo ${ }^{15}$.

Esta ola de capitales e inmigrantes en la Sierra Tecapa tuvo en la ciudad de Santiago de María el domicilio de la mayoría de ellos, incluyendo la proveniente de San Miguel, Usulután y Jucuapa que también contribuyó en un proceso que se extendió por todos los municipios de la zona. Pronto la ciudad de la inmigración se topó con la disposición cada vez más limitada de tierras para el cultivo del café, pues había muchos interesados en adquirirlas; por lo que la competencia entre éstos y la población local es un elemento importante en la configuración de los cafetaleros, algunos de los principales se presentan en el cuadro a continuación.

\begin{tabular}{|l|l|l|}
\hline \multicolumn{2}{|c|}{ Cuadro 2. Inmigrantes extranjeros e inversión cafetalera en Santiago de María, 1895-1915. } \\
\hline Nombre & Fincas, origen. & Otras actividades económicas. \\
\hline Prudencio & Administrador del Padre Lazplazas. & Beneficiador y exportador. \\
& $\begin{array}{l}\text { Compra en } 1907 \text { la mitad del } \\
\text { patrimonio de Lazplazas, recibe el } \\
\text { resto como herencia, la cual incluye }\end{array}$ & \\
& Miramar, vendida por Sol a Lazplazas. & \\
\hline Constantino & Compra entre 1899-1910 cerca de 20 & Comerciante, almacén de primera clase. A partir \\
& manzanas en la zona cotizada. Vende su & de la venta no se tiene ningún tipo de registro. \\
& patrimonio en 30,000 pesos a Evaristo & Probablemente retorna a Italia. \\
\hline
\end{tabular}

15 Pedro Fonseca. Prontuario geográfico y estadístico de la República de El Salvador. (San Salvador: Imprenta Nacional, 1915). p.158. 
Indexaciones: Repositorio de Revistas UCR, DIALNET, Latindex, REDALYC Directorio y recolector de recursos digitales del Ministerio de Cultura de España, Directory of Open Access Journals. Diálogos Revista Electrónica de Historia ISSN 1409-469X. Número especial 2008. Dirección web: http://historia.fcs.ucr.ac.cr/dialogos.htm

\begin{tabular}{|c|c|c|}
\hline $\begin{array}{l}\text { Benjamín } \\
\text { Guadamuz. }\end{array}$ & $\begin{array}{l}\text { Compra en } 1901 \text { la propiedad } \\
\text { embargada de Ezeta. Su hermano } \\
\text { adquiere otra finca de similar tamaño en } \\
\text { Alegría. }\end{array}$ & $\begin{array}{l}\text { Posee almacén de primara clase, 1901.EI patrimonio } \\
\text { es vendido luego de su muerte a Mauricio Meardi por } \\
\text { 30,000 pesos en 1911, incluye cerca de } 100 \text { manzanas } \\
\text { en la sierra y en tierra caliente. }\end{array}$ \\
\hline $\begin{array}{l}\text { Agustín } \\
\text { Montobbio }\end{array}$ & $\begin{array}{l}\text { Cafetales en Santiago de María, } \\
\text { Tecapán y California. Forma la finca } \\
\text { Sta. Catterina }\end{array}$ & $\begin{array}{l}\text { Habilitador, propietario de un hotel en Usulután. Tienda } \\
\text { de } 2 \text { da clase. Por dificultades económicas contrae } \\
\text { préstamos. Sus propiedades son ejecutadas hacia } 1917\end{array}$ \\
\hline Juan Mayner & $\begin{array}{l}\text { Compra paulatinamente cafetales, los } \\
\text { precios sugieren ser embargos a su } \\
\text { favor. }\end{array}$ & $\begin{array}{l}\text { Almacén de segundo orden, 1901. habilitador y } \\
\text { prestamista. }\end{array}$ \\
\hline Juan Homar & $\begin{array}{l}\text { Registros a partir de 1903, compras de } \\
\text { cafetales en } 3 \text { municipios. }\end{array}$ & Su esposa efectúa habilitaciones y préstamos. \\
\hline $\begin{array}{l}\text { Carlos } \\
\text { Amory }\end{array}$ & $\begin{array}{l}\text { Aparece en 1898. adquiere el } \\
\text { patrimonio del hermano de Guadamuz. } \\
\text { Fincas ubicadas en Alegría. Fincas en } 3 \\
\text { municipios por compras y embargos }\end{array}$ & $\begin{array}{l}\text { Desde } 1910 \text { es uno de los principal habilitadores de } \\
\text { café eran entregados en el beneficio San Luis, finca del } \\
\text { mismo nombre en Alegría }\end{array}$ \\
\hline $\begin{array}{l}\text { Emmanuel } \\
\text { Goenaga }\end{array}$ & $\begin{array}{l}\text { Fincas de considerables tamaños en } \\
\text { Tecapán y Santiago, por compras entre } \\
\text { 1905-1921. }\end{array}$ & $\begin{array}{l}\text { Posee beneficio en Tecapán. Ocas habilitaciones } \\
\text { registradas, otorga varios préstamos. }\end{array}$ \\
\hline Jaime Ferrer & $\begin{array}{l}\text { En } 1906 \text { compra la mitad de la herencia } \\
\text { de Simón Sol, finca de } 200 \mathrm{mz} \text { aprox. }\end{array}$ & Almacén de primera clase, 1901. \\
\hline $\begin{array}{l}\text { Baltasar } \\
\text { Ferreiro }\end{array}$ & $\begin{array}{l}\text { Compra terrenos formando fincas de } \\
\text { más de } 50 \mathrm{mz} \text { en Tecapán y Santiago. }\end{array}$ & Almacén de primera clase, 1901 \\
\hline
\end{tabular}


Otros inmigrantes españoles que se asentaron fueron Pedro Jijaume y el abogado Ricardo Midence. Se cuentan con sus referencias a partir de 1911 como agricultores de Santiago de María, compartiendo algunos patrones comunes de compra de pequeñas propiedades entre 3 y 5 manzanas. Hasta 1915 sus propiedades registradas no sobrepasan las 10 manzanas de extensión, por lo que su trayectoria probablemente se desarrolló en los años posteriores al periodo de estudio. Jinjaume realizó también algunas habilitaciones y préstamos a sus deudores en 1912 y 1917 que demuestra poseer capacidades de manejar capitales. Al igual que Juan Mayner, Midence se estableció con su esposa (también española) desde su tierra de origen a Santiago de María.

Se desconoce las conexiones establecidas entre estos inmigrantes para venir a El Salvador e invertir sus capitales como comerciantes, agricultores o agentes de créditos. Algunas de las relaciones identificadas son las de parentesco como las de Agustín Gotuzzo, uno de los pioneros quien llegó con sus esposa Colomba Canessa, luego Ambrosio Canessa (hermano de ella?) en la década de 1880. Otro caso es el Baltasar Ferreiro y su primo Francisco Mitjavila hacia 1908; el Presbítero Lasplazas y Prudencio Llach en las postrimerías del siglo XIX; los hermanos (o primos) Mauricio, Miguel y Francisco Meardi en los mismos años. La inmigración palestina y de otros puntos del Cercano Oriente, tiene su presencia con el arribo de varios comerciantes, entre ellos están Salomón Pedro Facdol (1901), Antonio Elías (1908) Elías Hasbún (1909), Santiago Yasbeck, Antonio Samour y Juan Salomé (antes de 1920).

Hacia inicios dela década de 1920 serealiza una especie de censo de extranjeros residentes en el municipio, donde se mencionan a casi todos los citados, entre otras personas aparentemente no establecidos permanentemente pero con nexos con los primeros; alcanzando una cantidad de 30 hombres. 
En lo que respecta a las estrategias económicas que les permiten convertirse en actores importantes del auge cafetalero están las habilitaciones a la pequeña producción cafetalera. En las inscripciones de esta actividad es claro que los beneficiadores de café como Carlos Amory, Mauricio Meardi y Mauricio Del'Pech son los principales actores del financiamiento de la pequeña producción cafetera, especialmente la de los municipios de Tecapán, Alegría y Santiago de María entre 1910 y 1917 registrando 50 y 35 habilitaciones respectivamente. El resto de habilitadores, entre extranjeros y locales, es bastante menor la proporción de su participación en esta área. Alberto Ávila, Miguel Ángel Gutiérrez y Rafael Benjamín Colindres son los actores locales con mayor participación en esta actividad. La participación de José Trabanino y la empresa de Salvador Mugdan \& Cía, empezó a producirse hacia 1915 y continuando fuera del periodo de estudio.

Es de destacar el funcionamiento de empresas agrícolas durante las décadas 1897-1917, pero muy pocas tuvieron éxito. Las sociedades Meardi-Del'Pech y la "Coffe Development Company of North America" representada por Amadeo Sesostris Canessa fueron sin duda las más importantes y exitosas por contar con fuertes recursos financieros, capacidad y estrategias por parte de sus representantes, así como también por sus conexiones con el exterior, entre otros factores. La primera comienza a funcionar en 1905 luego de una previa sociedad por parte de Del'Pech. La mayor parte de las propiedades de esta sociedad, se producen a partir de la compra de terrenos a personas naturales como también a sociedades extintas como la Prieto-Macay y la Grimaldi Hermanos, ambas de Tecapán. En Santiago de María, las principales adquisiciones provienen de las propiedades compradas a los sanmigueleños Victoriano Ruíz y Victoriano Flores. En resumen, son más de 150 manzanas en cada municipio los que forman el patrimonio de la sociedad Meardi-Del'Pech. 
La segunda se forma hacia 1908 con la finca de Ambrosio Canessa en Santiago de la finca llamada "La Veneciana", compuesta de 150 manzanas totalmente equipada para beneficiar café; así como otros terrenos hacia el oriente en la frontera con Jucuapa donde compró cerca de 40 manzanas en 1900. Las adquisiciones de mayor extensión se ubicaron en California, desde las 10 hasta 190 manzanas compradas entre 1900 y 1907 con las cuales formó la hacienda San Ambrosio (10 caballerías) ubicada en las alturas del volcán de Usulután compartido por los municipios de Jucuapa, California, Santa Elena y Ereguayquín ${ }^{16 *}$.

No menos importante es la migración de sanmigueleños, entre los cuales destacan algunos de los cafetaleros pioneros como lo son Simón Sol, Jaime Ávila Manuel Calvo y Leónidas Arguello, sumándose otros como Alberto Ávila que tuvo propiedades en Tecapán y residencia en Santiago de María. Victoriano Ruíz administró fincas en las faldas del Tigre y valle del mismo en el Norte del municipio de estudio. En esos dos puntos manejó fincas de 35 y 40 manzanas respectivamente a nombre del Banco Salvadoreño de quien recibió un crédito en 1896. En 1091 Ruíz hipotecó las mismas a favor de su acreedor por deudas de 30,000 pesos, por lo que recibe un refinanciamiento a dos años de plazo ${ }^{17}$. Al parecer la medida funcionó por varios años, pero en 1915 fue comprada la porción de 35 manzanas por la referida sociedad de Meardi $^{18}$.

En cuanto a los provenientes de mismo departamento de Usulután, están algunos miembros

16 CNR-Usulután. Libro 22 del Registro de Propiedad, partidas 46-56 y Libro 23 del Registro de Propiedad, partidas 79-86.

* De esta hacienda se formó el municipio de California en 1897 por instancias de Ambrosio Canessa, quien financió la construcción del cabildo e iglesia, cuyo patrón es el santo de su nombre. Santiago Barberena. Monografía. p.30.

17 CNR-Usulután. Libro 4 del Registro de Propiedad, partidas 272-274.

18 CNR-Usulután. Libro 39 del Registro de Propiedad, partida 337. 
de las familias Araujo, Samayoa, Guandique, Munguía, Zelaya y Montes quienes se trasladan desde sus localidades de origen (Usulután y Jucuapa) hacia alguno de los pueblos de la Sierra, principalmente a Santiago de María. Federico y Juan José Samayoa, por ejemplo, poseen la hacienda El Bongo en Tecapán, formando terrenos con café en el norte de la misma jurisdicción por parte del Dr. Salvador Samayoa hijo. Hacia 1910 los primeros miembros citados se asentaron en Santiago de María con la apertura de sus consultorios y farmacias, trasladándose el resto de la familia posteriormente.

Algunos miembros de la familia Araujo hacen lo mismo desde Tecapán, Jucuapa y Alegría. La de Tecapán estuvo emparentada con los Samayoa de la cual provienen los miembros mencionados. En cuanto a los de Alegría y Jucuapa, parece haber una relación según herencias y traspasos de propiedades en las mismas jurisdicciones y en Santiago de María donde tuvieron fincas de considerable extensión. Los principales caficultores de este grupo son el abogado Dr. Miguel Araujo y su hijo Rosendo, vecinos de Jucuapa y nacidos en Alegría. Sus fincas se ubicaban el los límites entre la primera y Santiago de María, probablemente desde los antiguos ejidos de Jucuapa donde una parte fue segregada para formar la segunda.

Como se ha expuesto con anterioridad, es posible determinar a los principales cafetaleros de Santiago de María por algunas características básicas en que se pueden agrupar. Los pioneros tuvieron la ventaja de formar propiedades durante la expansión cuando la disponibilidad y facilidad debido a la poca demanda y las formas de tenencia de la tierra como situaciones favorables para formarlas. Los comerciantes por su parte, la capacidad y el capital que les daba su oficio, fue un buen comienzo para incursionar, al igual que la actividad del crédito o préstamo 
que por lo general estaban en las manos de estos dos grupos. Los campesinos locales no tenían estas capacidades, pero si la posesión de las tierras. A medida que se fraccionan para cada uno de los descendientes, estos van vendiendo sus pequeñas parcelas, otros sus herencias, o fueron sujetos de hipotecas donde terminaron perdiéndolas a favor de sus acreedores, situación de la que tampoco escapan personas con mayores recursos.

Conformación de una micro región cafetalera en la Sierra Tecapa.

Es claro que los principales caficultores son de origen extranjero y de viejas familias propietarias de la Sierra Tecapa. Sin embargo, la competencia entre las grandes fincas con las pequeñas y dispersas de un mismo caficultor parece llevar a unos a la compra en otras zonas de la Sierra, principalmente en los municipios vecinos cuyo resultado se plasmó en la configuración de un poder económico-cafetalero que dio forma a una micro-región con su respectiva capital en el municipio de estudio.

Son dos grupos básicos de cafetaleros quienes tuvieron intereses de este tipo en la zona. Por una parte están los cafetaleros más importantes de Santiago de María con propiedades y residencia en la misma; por otra parte están aquellos vecinos de ésta (de diversos orígenes) con propiedades en jurisdicciones vecinas. En la anterior presentación de los extranjeros se pone referencia a los patrones geográficos de sus inversiones, por lo que es bastante fácil reconocer quienes son los principales cafetaleros del Distrito de Alegría o de Santiago de María. 
Indexaciones: Repositorio de Revistas UCR, DIALNET, Latindex, REDALYC Directorio y recolector de recursos digitales del Ministerio de Cultura de España, Directory of Open Access Journals. Diálogos Revista Electrónica de Historia ISSN 1409-469X. Número especial 2008. Dirección web: http://historia.fcs.ucr.ac.cr/dialogos.htm

\begin{tabular}{|c|c|c|c|c|}
\hline \multicolumn{5}{|c|}{ Cuadro 3. Principales cafetaleros de Santiago de María y en la Sierra Tecapa. } \\
\hline Caficultor & Tecapán & Alegría & Berlín & Otros municipios \\
\hline $\begin{array}{l}\text { Mauricio } \\
\text { Meardi }\end{array}$ & $\begin{array}{l}4 \text { caballerías en } 1904 \text {, } \\
\text { 150mz hipotecadas a su } \\
\text { favor en } 1905 \text {, } \\
\text { 16mz compradas en } 1908\end{array}$ & $\begin{array}{l}\text { 130mz por varias } \\
\text { compras que suman } \\
1,000 \text { pesos o más. }\end{array}$ & $\begin{array}{l}\text { Entre 1901-1917: } \\
420 \mathrm{mz} \text { en numerosas } \\
\text { compras por un total } \\
\text { de } 150,000 \text { pesos, } \\
\text { algunas reúnen } \\
140,000 \text { cafetos. }\end{array}$ & $\begin{array}{l}\text { 80mz en Santa Elena. } \\
\text { Porción de la } \\
\text { hacienda Santa Anita } \\
\text { en Mercedes Umaña }\end{array}$ \\
\hline Carlos Amory & $\begin{array}{l}\text { Inscribió a su favor } 21 \mathrm{mz} \\
\text { en } 1914 .\end{array}$ & $\begin{array}{l}\text { Entre } 1903-1914, \\
\text { alcanzado a comprar } \\
125 \mathrm{mz} \text {, reúnen } \\
45,000 \text { cafetos por } \\
\text { compras y embargos } \\
\text { a su favor }\end{array}$ & ----- & $\begin{array}{l}\text { 10mz en California } \\
200 \text { en San } \\
\text { Buenaventura entre } \\
1911-1914\end{array}$ \\
\hline $\begin{array}{l}\text { Agustín } \\
\text { Gotuzzo }\end{array}$ & $\begin{array}{l}15 \mathrm{mz} \text { con } 6,000 \text { cafetos } \\
\text { en } 1898 \text { y } 1903\end{array}$ & $\begin{array}{l}\text { Finca San Agustín, } \\
72 \mathrm{mz} .50 \text { de ellas } \\
\text { con } 40,000 \text { cafetos, } \\
1913 .\end{array}$ & -------- & ----------- \\
\hline $\begin{array}{l}\text { Evaristo } \\
\text { Galliano }\end{array}$ & $\begin{array}{l}\text { Finca El Caulote, } 127 \mathrm{mz} \\
\text { con beneficio de café, } \\
\text { por 40,000 pesos a León } \\
\text { Ávila }\end{array}$ & ------ & ------- & ----- \\
\hline $\begin{array}{l}\text { Emmanuel } \\
\text { Goenaga }\end{array}$ & $\begin{array}{l}\text { 112mz por compras y una } \\
\text { deuda a su favor }\end{array}$ & $\begin{array}{l}\text { 130mz, en parte con } \\
5,000 \text { cafetos. } 1905\end{array}$ & & \\
\hline
\end{tabular}


Las sociedades agrícolas como las de Mauricio Meardi y Ambrosio-Amadeo S. Canessa, constituyen una pieza clave de este fenómeno, según se ha expuesto anteriormente. Algunos cafetaleros medianos son Ester Cárdenas de Homar con 1 caballería en Ozatlán y la hacienda Prusia en Jiquilisco (10 caballerías); Baltasar Ferreiro con 40mz en Tecapán ${ }^{19}$, Raúl Ávila Bustamante quien adquirió la finca Esperanza en Berlín, con 30,000 $\operatorname{cafetos}^{20}$. Otros cafetaleros avecindados en Santiago de María como el suizo Samuel Wyler y los Samayoa (mencionados en el aparatado anterior), Benjamín Serpas, Salvador Ávila Bustamente y los abogados Modesto Castro, Rafael Benjamín Colindres, Salvador Cárdenas Rodríguez y Salvador Zelaya (farmaceuta) tuvieron propiedades en esta jurisdicción, como en Alegría, Berlín y Tecapán. Estas referencias son nada más el factor económico sobre el se fue configurando la microregión cafetalera de la Sierra Tecapa, específicamente el Distrito de Alegría, a partir de los intereses cafeteros en esta zona.

El auge cafetalero inició con el asentamiento de más inmigrantes, entre comerciantes y agricultores que formaron importantes patrimonios cafeteros. En segundo lugar estuvieron aquellos que, procedentes principalmente de Alegría y Jucuapa, adquirieron o registraron propiedades a su favor. Sin embargo, las ventajas de las habilitaciones, créditos y otros capitales facilitados por el comercio, o traídos desde el país de origen permiten a los extranjeros ubicarse como los cafetaleros más fuertes junto a la primera generación que continuó con similares patrones de concentración y ubicación de sus propiedades. A medida que la competencia por tierras en Santiago de María se agota, varios buscaron adquirir en los municipios vecinos, construyendo un territorio más o menos homogéneo en cuanto a la tenencia de la tierra. Cohesión y ejercicio

19 CNR-Usulután. libro 35 del Registro de Propiedad, partidas 366-367.

20 CNR-Usulután. libro 31 del Registro de Propiedad, partida 53. Año de 1911. 
de una territorialidad, se configuró en base a redes entabladas entre este grupo como se tratarán en las próximas páginas.

\section{Redes sociales: formando capitales sociales a través de enlaces matrimoniales.}

La generación nacida de los caficultores pioneros muestran interés de contraer matrimonio con los extranjeros avecindados en Santiago de María, o con personas provenientes de familias económicamente importantes tanto de la Sierra como de puntos más distantes, especialmente de San Miguel. Los hijos de los pioneros Juan Schonemberg y de Agustín Gotuzzo son los primeros en formar estas uniones según se presentan en el siguiente cuadro.

\begin{tabular}{|c|c|c|c|}
\hline \multicolumn{4}{|c|}{$\begin{array}{l}\text { Cuadro No.4. Matrimo } \\
\text { de Santiago de María }\end{array}$} \\
\hline \multicolumn{2}{|c|}{ La sucesión de Juan Schonemberg y Leonor Silva } & \multicolumn{2}{|c|}{ Hijos de Agustín Gotuzzo y María Teresa Canessa } \\
\hline Emma Schonemberg & $\begin{array}{l}\text { Prudencio Llach, uno de los } \\
\text { principales cafetaleros de } \\
\text { Santiago de María. }\end{array}$ & Agustín Gotuzzo hijo & $\begin{array}{l}\text { Fidelina Bustamante. De } \\
\text { San Miguel, hija de un socio } \\
\text { del Banco Salvadoreño. }\end{array}$ \\
\hline Emilia Schonemberg & $\begin{array}{l}\text { Prudencio Lach, cuando } \\
\text { enviudó de la primera. }\end{array}$ & Colomba Gotuzzo & $\begin{array}{l}\text { Emmanuel Goenaga, uno } \\
\text { de los cafetaleros del } \\
\text { municipio }\end{array}$ \\
\hline Elvira Schonemberg & $\begin{array}{l}\text { Evaristo Galleano, uno de los } \\
\text { cafetaleros del municipio }\end{array}$ & Paula Gotuzzo & $\begin{array}{l}\text { Antonio José Macay, } \\
\text { minero de San Miguel. }\end{array}$ \\
\hline
\end{tabular}




\begin{tabular}{|l|l|l|l|}
\hline Ana Schonemberg & $\begin{array}{l}\text { Juan Boillat, comerciante } \\
\text { suizo. }\end{array}$ & Elena Gotuzzo. & $\begin{array}{l}\text { Cap. Asencio Meléndez. Sin } \\
\text { más datos }\end{array}$ \\
\hline $\begin{array}{l}\text { Fuentes: Registros de propiedad a favor de algunos conyuges. } \\
\text { Diligencias matrimoniales, años de } 1902 \text { y } 1920 .\end{array}$
\end{tabular}

Otros casos se enmarcan en el contexto de las uniones matrimoniales entre inmigrantes e hijas de agricultores importantes de la Sierra Tecapa y Usulután como se aprecia a continuación.

\begin{tabular}{|c|c|}
\hline \multicolumn{2}{|c|}{ Cuadro No.5. Inmigrantes en Santiago de María y sus esposas salvadoreñas } \\
\hline Inmigrante avecindado & Esposa \\
\hline Juan Homar & $\begin{array}{l}\text { Ester Cárdenas, proveniente de priminente familia de Jucuapa. Hermana del } \\
\text { abogado Samuel Cárdenas, su hermana se casó con un inmigrante suizo. }\end{array}$ \\
\hline Baltasar Ferreiro & $\begin{array}{l}\text { Julia Gutiérrez, hija del hacendado añilero Vicente Gutiérrez, quien dejó } \\
\text { fincas a la sucesión en Santiago de María, Tecapán y California }\end{array}$ \\
\hline Manuel Vidaurre & Concepción Gutiérrez, hermana de Julia. \\
\hline Carlos Amory & $\begin{array}{l}\text { Hirlebranda Arias, hija de Perfecto Arias, agricultor y comerciante de } \\
\text { Alegría. }\end{array}$ \\
\hline Adrián Ortíz & Mercedes Rivera, de familia de agricultores de Santiago de María. \\
\hline Francisco Meardi & Rosa Ávila, hija del Gral. Jaime Ávila, caficultor pionero de Santiago. \\
\hline Pedro Jinjaume & Concepción Chavarría, de familia cafetalera local. \\
\hline
\end{tabular}

De la unión de capital extranjero con el social y agrario locales, surgieron importantes patrimonios cafeteros. Uno de estos casos es la dote de 30 manzanas en Alegría que don Perfecto Arias entregó a su hija cuando se casó con Carlos Amory. También se encuentra el ejemplo de Julia Gutiérrez quien compró parte de la herencia de su hermano Miguel Ángel, consistente en las 2/6 partes de la finca La Maravilla en Santa Elena, las cuales arrendó a su esposo Baltasar Ferreiro ${ }^{21}$. Uno de 21 CNR-Usulután. Libro 33 de propiedad, partida 239. 
los ejemplos más interesantes es el patrimonio de Mauricio Meardi y su espora Rosa Ávila, quien inscribió a su favor todas las propiedades que su esposo adquirió entre los años 1910-1917, más algunos terrenos heredados a ella por su padre (el Gral. Jaime Ávila) los cuales pasaron a formar la finca "Villa Rosita" situada en el Cerro Verde, al Suroeste de Santiago de María ${ }^{22}$.

En cuanto a Mercedes viuda de Ortiz y Ester Cárdenas de Homar, ambas fueron activas compradoras de tierras en el municipio de estudio. Este fenómeno no fue único de Santiago de María, pero si donde se produjeron más casos. Según algunas adquisiciones y traspasos en los Registros de Propiedad, las señoras Eva Araujo de Mongiardini, Elena Cárdenas de Schultz, e Irene Claros de Silis, formaron sendas uniones con extranjeros avecindados en Jucuapa, en la cual se formaron considerables patrimonios cafeteros.

A medida que la ciudad se convierte en un activo centro de comercio y actividades administrativas correspondientes como cabecera del Distrito de Alegría (conservó el nombre antiguo aunque la cabecera fue trasladada) recibió el asentamiento de abogados y profesionales de la medicina. Muchas de estas personas se establecen definitivamente en el municipio de estudio, algunos adquiriendo propiedades cafeteras o poseyendo por herencia familiar en ésta y en municipios de la zona. Algunos de estos hombres agregan la prestación de sus servicios profesionales como se muestran a continuación.

\begin{tabular}{|l|l|}
\hline \multicolumn{2}{|c|}{ Cuadro No.6. Sanmigueleños, usulutecos y familias de la Sierra Tecapa residentes en } \\
\multicolumn{2}{|c|}{ Santiago de María } \\
\hline Agricultor avecindado y origen & Esposa, origen \\
\hline Rosendo Araujo, Jucuapa-Alegría & Olimpia Colindres, Jucuapa \\
\hline
\end{tabular}

22 CNR-Usulután. Libro 47 de propiedad, partidas 138-143. 


\begin{tabular}{|l|l|}
\hline Miguel Ángel Araujo, Alegría & Marta Vides (sin datos) \\
\hline Raúl Francisco Munguía, Usulután. & María Elena Araujo, hija de Rosendo. \\
\hline Victoriano Ruíz, San Miguel & Arcadia Quirós de Ruíz, San Miguel \\
\hline Pablo José Aguirre, ingeniero topógrafo de San Miguel & Dolores Valenzuela de Aguirre, San Miguel \\
\hline Salvador Zelaya, farmaceuta de Jucuapa & Josefina Arguello de Zelaya, Jucuapa \\
\hline Francisco Monterrey, abogado (de San Salvador?) & Irene Sol, hija de Simón Sol. \\
\hline Rafael Benjamín Colindres, abogado. & Virginias Arias, Alegría. \\
\hline Modesto Castro, abogado de Alegría & Salvadora de Castro (sin datos) \\
\hline Miguel Ángel Gutiérrez, hijo de Vicente Gutiérrez & Mercedes Rivera (sin datos) \\
\hline Salvador Samayoa, farmaceuta de San Miguel & María Ignacia Araujo, Tecapán \\
\hline
\end{tabular}

Se han expuesto a los actores sociales (principalmente extranjeros, sanmigueleños y algunos jucuapanenses) que forman el grupo socio-económico del que se configuró la pequeña capital regional de Santiago de María. No cabe duda que la "cuantiosa inmigración" a la que se refiere Santiago Barberena y otros, tuvo un componente casi determinante en el fenómeno de la formación de la elite económica no solo del municipio de estudio, sino también del Distrito en el cual se enmarca la zona de estudio.

Las familias locales que forman parte de este grupo, fueron aquellas que desde la generación primera tuvo acceso a registrar considerables porciones a su favor, como los casos de los Mejía y Chavarría. Aun frente a las ventas de tierra de algunos de los miembros descendientes de dichas familias, hombres como Agapito Mejía y el Dr. Pedro Chavarría respectivamente, figuraron como prominentes cafetaleros de la localidad. Otros como Luis López, Miguel Rivas Garay y las 
señoras esposas de agricultores foráneos son parte de esta elite local.

El prestigio y el respaldo de cafetaleros y profesionales es importante en la formación del capital social entre la elite económica local, quienes además muestran interés por participar en asuntos de la vida local y en expresiones más ambiciosas como se expondrán en el siguiente apartado.

\section{Redes políticas: Vinculando intereses particulares, locales y regionales.}

\section{La municipalidad.}

Al comenzar el siglo XX, Santiago de María no es poblado agrícola más. Se trata de una zona cafetalera ya emergida donde la importancia de esta actividad se manifiesta no solo en la extensa superficie cultivada, en la inmigración o en las decisiones municipales sobre los impuestos como se ha expuesto en el tercer capítulo. La lectura de las actas municipales de este periodo refleja también un crecimiento de actividades y servicios en el área urbana como manifestación tanto de un crecimiento poblacional como también de la complejidad social y sus respectivas dinámicas socio-económicas.

Entre la complejidad ocasionada de este crecimiento destaca el problema de la escasez de agua y los intereses cafetaleros. Este caso es una de las primeras referencias sobre el interés de los cafetaleros en asuntos locales, pues se trata de la provisión de agua para sus fincas. Una de estas manifestaciones es la conformación de la "Junta de Agua" en 1892, un acto por tomar cartas en el asunto ante la incapacidad de fondos municipales y la dificultad de la intervención de autoridades gubernamentales para resolver el asunto. Los principales cargos directivos de dicha Junta fueron 
ocupados por los cafetaleros y, aunque el problema nunca fue lo suficientemente resuelto, es razón por la cual se realizaron numerosas peticiones y reuniones en el cabildo municipal registradas durante todo el periodo.

En esta misma década los cafetaleros prominentes comienzan a figurar en el control de la municipalidad. Según las actas, la nómina de Alcaldes Municipales de Santiago de María desde su fundación hasta finalizar el siglo XIX, estuvo compuesta por los pobladores cuyos nombres y apellidos corresponden a la lista de vecinos que acordaron la ubicación del emplazamiento urbano y otras decisiones relativas a la fundación y creación del municipio entre 1874-1876²3. Esta situación cambió cuando la actividad del café despega como la principal actividad económica y fuente de ingresos municipales. Algunos caficultores locales como el Dr. Ramón Araujo, el hacendado añilero Vicente Gutiérrez y Luis López presidieron el gobierno local, mientras que en los consejos municipales figuran nombres como Simón Sol, Agustín Gotuzzo y Alberto Ávila como síndico y regidores en 1891 y $1892^{24}$.

La calificación de negocios y el ordenamiento del comercio en las calles para aumentar las rentas municipales, así como ornato e higiene fueron impulsadas durante las administraciones conducidas por los primeros profesionales de la localidad, tales como los doctores Manuel Recinos, Ramón Araujo, Francisco Cárdenas Rodríguez, César y Modesto Castro entre 1895-1901, originarios de municipios vecinos (cuadro 6). A partir de esta fecha, los concejos municipales estuvieron conformados por cafetaleros, comerciantes y profesionales locales avecindados (y desde entonces

23 AMSM. Libro de Actas Municipales de 1875 y 1876, sesiones del $1^{\circ}$ de junio y 10 de julio, respectivamente.

24 AMSM. Libro de Actas Municipales de 1891 y 1892. Primeras sesiones de ambos años. 
santiagueños) como los siguientes:

\begin{tabular}{|l|l|l|}
\hline Año & Alcalde Cuadro No.7. & Concejos Municipales de Santiago de María, 1901-1917. \\
\hline 1901 & Dr. Rafael B. Colindres & Pablo Lara, Leonidas Arguello, Victoriano Ruíz, Joaquín Loucel \\
\hline 1903 & Victoriano Ruíz & Juan Miguel Cora, Alberto Ávila, Dolores Montoya, Salvador Samayoa \\
\hline 1905 & Juan M. Cora & Pablo Lara, Salvador Samayoa, Dr. Modesto Castro \\
\hline 1906 & Victoriano Ruíz & Dr. Salvador Zelaya, Armando Ávila, Simón Araujo \\
\hline 1907 & Juan Miguel Cora & Dr. Fernando Mejía, Dr. Daniel Quirós Ruíz, Pablo Lara \\
\hline 1908 & Luis López & Salvador Samayoa, Baltasar Ferreiro, Dr. Pedro Chavarría, \\
\hline 1910 & Dr. Salvador Zelaya & Emmanuel Goenaga, Emilio Cáceres, Miguel Á. Gutiérrez, Dr. José Zelaya \\
\hline 1911 & Dr. José Estrada Colindres & Alfonso Zelaya, Juan José Samayoa, Salvador Ávila y Dr. César Castro \\
\hline 1912 & Carlos Amory & Miguel Rivas Garay. Sin más datos. \\
\hline 1914 & Carlos Amory & Doctores Leonidas Ávila, Samuel Cárdenas, Rafael Castillo; M. Chavarría \\
\hline 1915 & Dr. Rafael B. Colindres & Carlos Duke, Dr. Fernando Mejía, Salvador Padilla \\
\hline 1917 & Carlos Amory & Francisco Meardi, Rafael Samayoa, Ezzio Montobbio, Enrique Araujo \\
\hline
\end{tabular}

Al comparar los nombres que integraron los concejos municipales con los cuadros elaborados de la migración en capítulos anteriores, es claro que el asentamiento de comerciantes, agricultores y profesionales no se limitó al ejercicio de sus ocupaciones. Esta tendencia parece mantenerse hasta la década de 1930, pues los alcaldes posteriores al periodo de estudio fueron algunos regidores anteriores como los cafetaleros Raúl Ávila (1919, 1930), Agustín Gotuzzo (1918, 1928), Miguel Ángel Gutiérrez (1924) y Amadeo Sesotris Canessa (1921).

Durante estos gobiernos municipales se llevaron a cabo discusiones, proyectos y medidas de interés y beneficio local, como también otros cercanos a los intereses de los gestores. Dentro del quehacer de estos concejos a destacar, están aquellas sobre el problema del agua, asuntos financieros, nombramientos de Juntas de Fomento, obras públicas y otras decisiones donde la 
elite local tuvo un papel importante en la toma y ejecución de las mismas.

Asuntos de escala geográfica mayor tratados por esta municipalidad son el Puerto El Triunfo y el uso de los fondos municipales del Distrito de Alegría. Según la serie de Actas Municipales con que se cuenta para el siglo XX, en la lectura de éstas se encuentra una de las primeras decisiones de la micro-región cafetalera, es decir el distrito de Alegría; tales como la convocatoria de 1903 del cabildo de Santiago a los concejos municipales de la zona para emitir un pronunciamiento contra el Decreto Legislativo sobre la entrega de los fondos de caminos del distrito al de Usulután, para financiar los edificios públicos de la cabecera departamental. Con el argumento de que el distrito necesita de los fondos para las vías de comunicación dentro del mismo, la reunión acordó enviar una solicitud a las autoridades superiores para evitar la pérdida de los fondos del Distrito.

En la misma convocatoria se trató la necesidad de utilizar dichos fondos (mas un subsidio por parte del Poder Judicial) para ampliar las funciones del juzgado, "siendo este distrito en la parte judicial el que más movimiento tiene en la zona de oriente, de lo cual está al corriente el Supremo Tribunal de Justicia..." razón por la cual se decidió recurrir a los diputados del departamento: Joaquín Loucel, Pedro Chavarría, (vecinos y concejales anteriores Santiago de María) y el Dr, Manuel Morales para que presentaran al congreso dicha petición ${ }^{25}$.

Dentro de este ramo, el concejo de 1906 acordó solicitar a los Poderes Legislativo y Ejecutivo, gravar con 12 y medio centavos el quintal de café cereza, oro o pergamino cosechado en Alegría, Berlín y California, para la apertura de carretera entre la ciudad de Santiago y las dos primeras ${ }^{26}$.

25 AMSM. Libro de Actas Municipales de 1903. Sesión del 21 de marzo.

26 AMSM. Libro de Actas Municipales de 1906. Sesión del 22 de febrero. 
El gravamen municipal sobre la actividad del café fue un tema con muy poca discusión en los concejos municipales representados por este sector. Las pocas intervenciones sobre el asunto son, junto a la anterior, la moción del regidor y cafetalero Salvador Zelaya en aumentar a 200 pesos mensuales el impuesto a los beneficios de café durante la temporada de cosecha entre noviembre y abril ${ }^{27}$. Sin embargo, el tema es escasamente tratado en las sesiones, sumado a la falta de una serie de libros de ingreso que no permiten plantear más sobre este delicado asunto para los intereses cafetaleros.

Es claro al parecer, que los recursos financieros de la alcaldía son insuficientes, ya que la juramentación de Juntas de Fomento provisionales y específicas estaban conformadas por las mismas personas: miembros de la elite local. El siguiente cuadro resume la participación de este grupo en las necesidades de la población.

Cuadro 8. Juntas y asociaciones de fomento de Santiago de María, 1906-1917

\begin{tabular}{|l|l|l|l|}
\hline Año & Motivo & Integrantes & Actas Municipales \\
\hline 1907 & Junta de Fomento & $\begin{array}{l}\text { Carlos Amory, Dr. Rafael Colindres, Juan y Rodolfo } \\
\text { Schonemberg, Armando Ávila y J.J. Samayoa }\end{array}$ & $\begin{array}{l}\text { Revista Municipal, } \\
\text { año 1, No.2 }\end{array}$ \\
\hline 1906 & $\begin{array}{l}\text { Construcción del } \\
\text { parque }\end{array}$ & $\begin{array}{l}\text { Colomba de Goenaga, Virginia de Colindres, Julia de } \\
\text { Ferreiro, Josefina de Zelaya, Salvadora de Castro, Arcadia } \\
\text { de Ruíz. Señoritas Mejía, Harrison, Schonemberg, Samayoa } \\
\text { y Araujo. }\end{array}$ & Sesión 5 de enero \\
\hline 1909 & $\begin{array}{l}\text { Construcción de } \\
\text { templo parroquial }\end{array}$ & $\begin{array}{l}\text { O. Luria, Luis Manent, Agustín Montobbio, Juan Homar, } \\
\text { Carlos Amory, Baltasar Ferreiro, Constantino Cocchella, } \\
\text { Pbto. Lasplazas, Dr, Modesto Castro, y otros. }\end{array}$ & 24 de febrero \\
\hline 1910 & $\begin{array}{l}\text { Junta de } \\
\text { Honorables }\end{array}$ & $\begin{array}{l}\text { Prudencio Llach, Dr. José Estrada Colindres, Casimiro } \\
\text { Donnadieu (tesorero), fondos recolectados para templo }\end{array}$ & Sesión 12 de enero \\
\hline
\end{tabular}

27 Idem. Sesión del 5 de enero. 


\begin{tabular}{|l|l|l|l|}
\hline 1911 & Junta de Festejos & $\begin{array}{l}\text { Dr. Rodolfo Schonemberg, Leonidas Ávila, Emilio Cáceres, } \\
\text { Pablo Scheidegger }\end{array}$ & Sesión 5 de julio \\
\hline 1912 & Junta de Fomento & $\begin{array}{l}\text { Carlos Amory, Emmanuel Goenaga, Casimiro Donnadieu, } \\
\text { Baltasar Ferreiro, Juan José Samayoa, Dr. B. Estrada }\end{array}$ & Sesión 12 de enero \\
\hline 1914 & $\begin{array}{l}\text { Comité de } \\
\text { Festejos }\end{array}$ & $\begin{array}{l}\text { Prudencio Llach, Mauricio Del'Pech, Ing. Pablo Aguirre, } \\
\text { Francisco Meardi, Agustín Gotuzzo, Ezzio Montobbio, } \\
\text { Juan M. Cora, Salvador Ávila, Dr. Rafael Samayoa. }\end{array}$ & Sesión 22 de junio \\
\hline
\end{tabular}

El mantenimiento de los caminos recibía un buen aporte de los vecinos "notables" como solían llamarlos en las actas municipales. Los contribuyentes de estos fondos eran cafetaleros y principales beneficiados de estas obras, pues de esa forma podrían transitar por buenos caminos las carretas cargadas con café provenientes de sus fincas ubicadas en los municipios del Distrito de Alegría.

La magnitud de la colaboración de las juntas de ayuda, fomento o beneficencia, según el nombre, la finalidad y los integrantes que pertenecían al mismo grupo, fue indispensable. Esta situación favorecía la creación de la figura pública o política gracias a sus redes clientelares y de apoyo, una práctica muy frecuente desde el siglo XIX. Sin duda alguna, durante el periodo de estudio esta figura fue el caficultor Carlos Amory, quien además de ocupar por tres años la silla edilicia, fue diputado por del departamento usuluteco en 1912, 1915 a 1917.

De lo expuesto con anterioridad, las asociaciones en pro del abastecimiento de agua y la mejora de los caminos, eran asuntos locales en los cuales se vieron más involucrados la elite cafetalera, comercial y profesional, no como grupos separados o distintos, sino como la composición de un grupo más o menos cohesionado por los intereses cafeteros, ya que varios comerciantes y profesionales eran también productores del grano rojo. 
Puerto El Triunfo, un proyecto a prueba de intereses particulares.

El mapa económico nacional en los albores del siglo XX se está reconfigurando, repercutiendo en la orientación y prioridad de las obras públicas. Los discursos de modernidad y progreso material se materializan en la medida que el Estado salvadoreño se fortalece políticamente gracias a una relativa estabilidad, mientras que en el plano económico se da a medida del auge de las exportaciones de café desde el último cuarto del siglo XIX. En este contexto comienza el proceso de la conectividad nacional a través del ferrocarril, que une al puerto de Acajutla con la ciudad de Santa Ana y de ésta con la capital entre 1894-1900. Entre esos años el proyecto desea llevarse a cabo en el otro extremo del país, con el ideal de atravesar el oriente salvadoreño y conectarse San Salvador.

El ferrocarril trans-oriental tiene un proceso de construcción lento, donde las obras padecen de constantes atrasos, cambios, suspensiones e incluso abandono. No ha sido posible aun determinar las razones de este lento y dificultoso proceso de construcción ferroviaria. No obstante, se tiene conocimiento de un proyecto alternativo para conectar la producción con destino al mercado exterior, una iniciativa por parte de empresarios agrícolas lo suficientemente fuerte como ambiciosa, esta es la habilitación del Puerto El Triunfo en la Bahía de Jiquisco, departamento de Usulután. 
El proyecto del Triunfo se enmarca en el despunte de la producción cafetalera del departamento de Usulután entre 1880-1890, donde la inversión de capitales extranjeros tiene un papel fundamental, principalmente en el municipio de Santiago de María, asiento de la segunda concentración de inmigrantes extranjeros en el oriente salvadoreño (después de San Miguel). En las primeras décadas del siglo XX, este departamento se ubica entre los 3 principales productores de café en el país, junto a los de Santa Ana y La Libertad; algunos de los principales productores del municipio mencionado figuran así mismo entre los principales exportadores de la nación.

Desde esta ciudad, el proyecto portuario tiene dos fases identificadas de vida. La primera se desarrolla en el último lustro del siglo XIX donde los principales caficultores como Mauricio Meardi, Ambrosio Canessa, Lorenzo Campos, Leonidas Argüello y Juan Schonemberg ${ }^{28}$ son accionistas de la Compañía del Triunfo Limitada, fundada en 1894 por iniciativa de empresarios estadounidenses. La cercanía de la Sierra usuluteca a la bahía donde se localiza El Triunfo respecto a La Unión es claramente conveniente para los productores que visualizan costos menores de fletes hacia el puerto, tiempos cortos de viaje de dichos fletes (lo que posibilita seguridad de la mercadería) así como la oportunidad de aprovechar la condición de exportador e importador al ser accionistas del puerto. Es importante mencionar que entre los accionistas y otros cafetaleros de Santiago de María poseen las conexiones necesarias con el mercado internacional, al grado de figurar entre los principales exportadores del país en los años de estudio.

Después de una efímera y dificultosa operación entre 1895-1900 la junta se declara en quiebra, la cual dejó entrever 3 serias dificultades: la poca profundidad de la bahía para la navegación de 28 AGN. Idem. p.15. 
buques de gran tonelaje, la poca capacidad financiera de la Compañía y el volumen insuficiente de mercancía e intercambio para sustentar en un plazo favorable la recuperación de la fuerte inversión del proyecto.

Hacia la segunda década del Siglo XX se verifica una segunda etapa de vida, la cual es el resultado de los cafetaleros santiagueños que han usado el poder político local para lograr la habilitación por parte de un contratista salvadoreño. Uno de los aspectos a remarcar de este estudio es la disputa en ambos periodos de los intereses económicos entre los cafetaleros de Santiago de María y algunos miembros del comercio sanmigueleño que vieron afectados sus negocios en el puerto unionense debido al uso alternativo del Triunfo.

Durante la administración municipal de 1903 que preside un importante cafetalero, Victoriano Ruíz, se convoca una sesión extraordinaria a las municipalidades del distrito, más vecinos propietarios del departamento, con el propósito de tratar la apertura del puerto. “Teniendo informes que se encuentran actualmente en San Salvador, una comisión compuesta de varios vecinos de San Miguel que so pretexto de la empresa del Ferrocarril central, pretenden que este puerto no sea reabierto, obligando así a los agricultores de la rica zona cafetalera de este departamento a exportar su café por el lejano puerto de La Unión, a costa de inmensos sacrificios.... y que la infundada pretensión de la comisión migueleña no tiene otro objeto que el deseo de sacrificar esta fracción laboriosa de la sección oriental, en provecho de sus representados. Si quieren ferrocarril, que lo hagan en hora buena, los usulutecos serán los primeros en aplaudir esa nueva

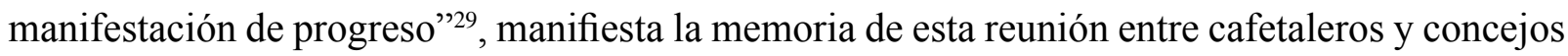
municipales quienes acordaron la conformación de una comisión compuesta de un representante 29 AMSM. Libro de Actas Municpales de 1903. Sesión del 29 de Septiembre. 
de cada municipio de este distrito para exponer al Presidente de la República sobre la necesidad de reapertura del Triunfo.

En el segundo periodo de Ruíz en la jefatura edilicia y del distrito, el concejo comisiona a Lorenzo Campos y Ambrosio Canessa con la misión de gestionar ante el gobierno sobre dicho asunto, con el propósito de beneficiar a los cafetaleros en la exportación de la próxima cosecha "que promete estar muy buena", acordaron los miembros de la junta ${ }^{30}$. Así mismo el concejo municipal de 1908 compuesto totalmente por cafetaleros, se reúnen con los cultivadores más importantes del departamento de Usulután, tales como Mauricio y Francisco Meardi, Francisco Lasplazas, Juan Cora, representantes de la sociedad Meardi y del Pech, Salvador Zelaya, Pablo José Aguirre, Evaristo Galliano, Benamín Guadamúz, Rodolfo Schonemberg, Ambrosio Canessa, Agustín Gotuzzo, Armando Ávila, Lorenzo Campos, Dr. Pedro Chavarría, Agapito Mejía, Salvador Samayoa, Carlos Amory, Benjamín Serpas, Antonio Araujo, entre otros 15 o 20 posibles cafetaleros para firmar una petición al gobierno central sobre la reapertura del Triunfo.

Tras años de gestiones entre cafetaleros de Santiago de María, alcaldes y diputados, la reapertura se ve realizada en el mes de octubre de 1911 durante la administración del Presidente Manuel Enrique Araujo, originario de Alegría. Meses después fue recibido con gran júbilo en Santiago de María, donde es recibido y hospedado por las personas más prominentes de la localidad ${ }^{31}$.

Sin embargo, el movimiento portuario del Triunfo en los años siguientes es bastante irregular. Mientras las visitas de vapores al Triunfo son muy esporádicas, limitándose al periodo de

$30 \quad$ AMSM. Libro de Actas Municpales de 1906. Sesión del 30 de abril.

31 AMSM. Libro de Actas Municipales de 1912. Sesión del 5 de julio. 
cosechas, el proyecto de la línea férrea oriental avanza finalmente y concreta algunos tramos de forma menos lenta y dificultosa. En el informe gubernamental del año 1911 se anuncia de la nueva contrata para terminar el ferrocarril entre San Miguel y el puerto de La Unión, que en ese momento está pendiente un corto tramo entre el río Grande y la ciudad ${ }^{32}$. En los años siguientes el proyecto fue tomado por la compañía Ferrocarriles Internacionales de Centro América (IRCA por sus siglas en inglés) que termina dicho tramo en 1912. En 1918 se concluye la línea de San Miguel-Zacatecoluca, pasando por el litoral de Usulután, mismo año donde no se verifican más vapores hacia puerto El Triunfo.

Cuatro años más tarde, (1922) finalmente el oriente y centro se comunica con la ciudad capital. La conectividad oriental se concreta en el mismo año con la construcción del puerto de Cutuco por la misma empresa del ferrocarril (a pocos kilómetros y en sustitución de La Unión) ambas estructuras satisfacen las necesidades de comunicación entre las zonas cafetaleras y los puertos. El puerto el Triunfo entonces, no registra más movimiento de buques, al menos para la exportación de café, quedando en servicio para la pesca artesanal.

\section{Conclusiones.}

La formación del poder local tiene entre sus componentes, los expuestos sobre la migración extranjera e interregional, como también los planteados sobre el cultivo, procesado y comercialización del café. Profesionales, comerciantes (varios de ellos con propiedades cafeteras) y cafetaleros compartieron un interés por los asuntos locales, en el marco del funcionamiento 32 AGMSS. Diario Oficial, tomo 72 No.44. 21 de febrero de 1912. 
de la gestión y las competencias que corresponden al gobierno local, caracterizadas por su autonomía respecto al central, pero dependiente de la voluntad de los alcaldes y de colaboración de los vecinos en la directa e indirecta ejecución de las obras públicas.

Redes sociales tejidas como las uniones matrimoniales entre los grupos que componen el poder económico y los profesionales; así como las asociaciones de fomento y los concejos municipales integrados por miembros de este grupo, constituyen los elementos de formación de un poder local sólidamente respaldado por el poder y los intereses cafeteros ejercidos desde la pequeña capital de la micro región cafetalera del Distrito de Alegría en la Sierra Tecapa, un fenómeno que probablemente no sea el único si se contase con estudios similares de otros municipios cafetaleros en occidente y centro para plantear patrones en las variantes locales.

La legitimidad y la base social de los conejos municipales escapan a lo límites de la investigación ante la falta de fuentes como actas y padrones electorales. Sin embargo, es claro que desde Santiago de María, los cafetaleros vecinos presionados por el agotamiento del espacio de cultivo en este municipio, extendieron sus intereses hacia los vecinos como Tecapán, Alegría y California, formando una territorialidad no solo administrativa sino también económica como Distrito.

Es importante conocer la trayectoria de estas redes desde la década de 1920 en adelante, cuando se acentúan fenómenos como la concentración de tierras, el aumento del territorio cafetalero que se expande hacia tierras de menor altura, conocer de manera más cercana las redes políticas en lo que respecta al ejercicio electoral, así como también profundizar en otros aspectos sociales que han escapado a la investigación por diversos motivos; pero se ha sentado un precedente en estudios 


\section{OI 9० CONGRESO \\ - $:$ CENTROAMERICANO \\ DE HISTORIA}

Universidad de costa Rica

Indexaciones: Repositorio de Revistas UCR, DIALNET, Latindex, REDALYC Directorio y recolector de recursos digitales del Ministerio de Cultura de España, Directory of Open Access Journals. Diálogos Revista Electrónica de Historia ISSN 1409-469X. Número especial 2008. Dirección web: http://historia.fcs.ucr.ac.cr/dialogos.htm

históricos locales y se necesita la continuación de este mismo y el inicio de otros para revisar y repensar la historia salvadoreña vista desde lo local, no como un cambio de planteamiento, sino de escala, ya que lo local está estrechamente vinculado con lo nacional y viceversa, aunque no siempre se recuerda esta visión. 